dem Bundeskanzleramt. Doch das ist kein Nachteil. Durch den engen Untersuchungsfokus auf die tatsächlichen (oder vermuteten) Querschnittsstrategen in den Landesregierungen gelingt Schwickert eine sehr detailgesättigte Analyse der Handlungsmöglichkeiten und -instrumente. Auf der Basis von zehn leitfadengestützten Interviews werden das Selbstverständnis der Planer, die jeweiligen Organisationszuschnitte sowie ihr Instrumentenkasten anschaulich gemacht. Durch die zugesicherte Anonymisierung ihrer Einschätzungen geben die Interviewpartner einen sehr tiefen Einblick in ihre Arbeit. Gleichwohl ist zu vermuten, dass sie nicht alle Karten auf den Tisch legen, insbesondere im Hinblick auf die zu unterstellende parteipolitische Überformung ihrer Arbeit (etwa in Wahlkämpfen).

Durchweg alle Interviewpartner berichten von einem Arbeitsalltag, in dem verwaltungstechnische Routinevorgänge und behördeninterne Abstimmungen die Planung und Strategieberatung überlagern. Einem strategischen Masterplan für das Regierungshandeln während einer Wahlperiode (oder darüber hinaus) steht die von allen Akteuren beschriebene Kurzatmigkeit des politischen Betriebs entgegen. Für Strategie bleibe im medial beschleunigten Alltagsgeschäft, so der Tenor der meisten Interviewten, kaum Zeit.

Schwickert schließt auf strukturell unzureichende Rahmenbedingungen für eine strategisch ausgerichtete Regierungspolitik und stellt fest, dass strategische Beratung, um durchsetzungsfähig zu sein, nicht nur das verwaltungstechnische Handwerk beherrschen, sondern eben auch das Ohr des Regierungschefs finden muss. Für eine Ausschöpfung ihrer kreativen und intellektuellen Potenziale fehlten den Planern in der Regel Zeit und Kapazitäten. Letztlich seien sie Planer nichts anderes als privilegierte, gut informierte, aber arbeitsüberlastete Verwaltungsmitarbeiter. Den interessierten Laien überraschen diese Thesen ebenso wenig wie den Eingeweihten, dem es allerdings an Zeit mangeln dürfte, dieses lesenswerte Buch aufzuschlagen.

Danko Knothe

\title{
Viele unverbundene Perspektiven auf Politik und Marketing
}

Alemann, Ulrich von, Martin Morlok und Julian Krüper (Hrsg.): (Partei-)Politik im Zeichen des Marketing (Schriften zum Parteienrecht und zur Parteienforschung, Band 40), Nomos Verlagsgesellschaft, Baden-Baden 2010, 145 Seiten, € 29,-.

Der vorliegende Sammelband ist der Ergebnisbericht des gleichnamigen Symposions, das am 18. und 19. April 2008 in Düsseldorf stattfand. Ausrichter war das Institut für Deutsches und Europäisches Parteienrecht und Parteienforschung. „Heute betreten wir Neuland, denn wir nehmen eine dritte Bezugswissenschaft hinzu, die Ökonomie. Heute sind wir nicht ein bi-disziplinäres Institut, sondern wir begehen ein tri-disziplinäres Symposion“ (S. 9), schreibt Ulrich von Alemann in der Einführung. Bereits der Titel des Buches verweist auf zwei der interdisziplinären Betrachtungsweisen, über die von Alemann referiert. Zum einen wird der Kernbereich der Politikwissenschaft in Form der (Partei-)Politikforschung angesprochen. Zum anderen wird der Begriff Marketing als einer der Grundpfeiler der Wirtschaftswissenschaft in die Diskussion eingebracht. Die dritte Dimension gewinnt das Symposion durch die inhaltliche Ausrichtung des Institutes für Deutsches und Euro- 
päisches Parteienrecht und Parteienforschung, das nicht auf die genuin politikwissenschaftliche Sphäre beschränkt ist, sondern auch eine juristische Perspektive verfolgt.

Der interdisziplinäre Ansatz des Sammelbandes erweist sich als seine größte Stärke und birgt gleichzeitig seine größte Schwäche. „Wir haben uns bemüht, allen drei Wissenschaften gerecht zu werden und durch prominente Referenten präsentieren zu lassen“, konstatiert von Alemann (S. 9). Dieser Aussage ist beizupflichten; in der Tat gibt das Buch einen umfangreichen Überblick verschiedenster Ansätze in unterschiedlichen Disziplinen: von Marketingaspekten in der Politik (Helmut Schneider, S. 12 ff.), über die juristische Betrachtung der Öffentlichkeitsarbeit der Regierung (Christoph Engel, S. 42 ff.), bis hin zur ideengeschichtlichen Abhandlung des Warencharakters von Politik (Guy Kirsch, S. 105 ff.). Das Spektrum ist breit und die Beiträge hinsichtlich Inhalt und Methode sehr verschieden. Das klingt in erster Linie positiv, allerdings ist dieser Eklektizismus nicht immer sinnstiftend, und so ergeben sich aus der wenig systematisierten Zusammenstellung des Bandes zwei Kritikpunkte:

(1) Statt einer interdisziplinären Betrachtung, die eine fächerübergreifende Verschränkung verlangt, werden die drei Ebenen Rechtswissenschaft, Politikwissenschaft und Wirtschaftswissenschaft losgelöst voneinander abgehandelt. Für einen innovativen Mehrwert, der in einer Verknüpfung zweier oder sogar aller drei Perspektiven bestehen könnte, sind die Kapitel zu disziplintreu und wagen nicht den Blick über den Tellerrand. Sie stehen für sich und verharren größtenteils bei den selbstgesetzten Themen. Die individuelle Güte einiger Beiträge soll damit in keinem Fall bestritten werden, allerdings muss der Mehrwert des Buches als Zusammenfassung der Thematik „(Partei-)Politik und Marketing“ kritisch betrachtet werden. Der Titel „(Partei-)Politik im Zeichen des Marketing“ findet sich zum Beispiel in dem juristisch fundierten und gut strukturierten Text von Matthias Rossi zur rechtlichen Absicherung der innerparteilichen Willensbildung nicht mehr wieder (S. 79). Über eine gelungene deduktive Herleitung, die bei verfassungsrechtlichen Minimalanforderungen des Art. 21 GG beginnt, gelangt Rossi zwanzig Seiten später zum speziellen Fall des „präventiven Zulassungsvorbehalts für Satzungen“ (S. 99). Hier, wie an einigen anderen Stellen auch ${ }^{1}$, ist der Bezug zum zentralen Thema fragwürdig. Es ist keine interdisziplinäre Herangehensweise zu erkennen, sondern fachspezifisches Detailwissen. Die stark differierenden Schwerpunkte der einzelnen Beiträge und die verschiedenen inhaltlichen und methodischen Stoßrichtungen lassen im Gesamtbild leider keinen roten Faden erkennen.

(2) Der zweite Kritikpunkt ist mit der Auffassung von Marketing verknüpft. Der Grund dafür ist im unscharfen Umgang mit dem Begriff zu finden. Zwar gibt von Alemann in seiner Einführung eine brauchbare Definition vor (S. 9), die sogar die verschiedenen Dimensionen des umfangreichen Marketingbegriffs berücksichtigt; leider wird diese aber meistens sträflich ignoriert oder gänzlich ausgespart. Im längsten Beitrag des Buches (Christoph Engel, S. 42 ff.) findet Marketing nicht einmal Erwähnung. In anderen Kapiteln wird der Begriff fälschlicherweise auf einzelne Dimensionen reduziert, so bei Hubert Kleinert (S. 129 ff.), der Marketing anfangs auf Werbung verkürzt und dann eine Abhandlung über die Gefahren der Mediatisierung der Politik schreibt. Ähnliches gilt für Patrick Donges (S. 30 ff.), der in seinem Beitrag über Professionalität den Marketingbegriff zwar als Alibi in der

1 Siehe auch die Beiträge von Christine Hohmann-Dennhardt (S. 70 ff.) zur Delegationsfähigkeit der politischen Willensbildung, sowie Christoph Engel (S. 42 ff.) zur ökonomischen Theorie in der Rechtswissenschaft. 
Überschrift erwähnt, im Text allerdings keinen Bezug mehr dazu herstellt. Diese begriffliche Problematik ist auch den Teilnehmern des Symposions nicht verborgen geblieben; Martin Morlok konstatiert völlig zutreffend im Schlusswort: „Daneben haben wir Marketing die letzten zwei Tage immer auch als einen unscharfen Begriff der Verkaufstechniken, der Selbstdarstellungsmethoden, der Werbung verwendet." (S. 142)

Ein Fazit könnte positiv formuliert lauten: Dieses Buch bietet facettenreiche Beiträge, die sich in der Summe nicht thematisch beschränken lassen. Das negative Pendant hieße: Der Großteil der Beiträge verfehlt das Thema, das der Titel verspricht. Die Wahrheit liegt in der Mitte. Die Güte dieses Bandes liegt in der Summe der Einzelbeiträge. Diese sind zum größten Teil fachlich sauber, gut strukturiert und in sich geschlossen. Die Summe, die sich daraus ergibt, ist weniger ergiebig, weil ein sinnvoller Transfer von der Beitragsebene auf das Gesamtwerk nicht gelingt. So findet man wenig neue Ansätze, die eine interdisziplinäre, also verschränkende Perspektive einnehmen. Wer dieses Buch liest, bekommt keinen zusammenhängenden Überblick zur „(Partei-)Politik im Zeichen des Marketing“. Insgesamt also ein solides Werk mit einer großen Bandbreite an Beiträgen, die lediglich in einer losen Verbindung zueinander stehen.

Felix U. Till

\title{
Wichtiger Beitrag zum Einfluss der Medien auf politische Unzufriedenheit
}

\author{
Floß, Daniela: The Impact of Mass Media on Political Support. A Preference-Perceptions Model \\ of Media Effects (Politische Kommunikation und demokratische Öffentlichkeit, Band 1), Nomos \\ Verlagsgesellschaft, Baden-Baden 2010, 218 Seiten, € 29,-.
}

Um dauerhaft überleben zu können, benötigen politische Systeme die Unterstützung ihrer Bürger. Bevölkerungsumfragen belegen jedoch, dass diese politische Unterstützung in nahezu allen westlichen Demokratien seit Jahrzehnten sinkt. Seit der Entdeckung des Zusammenhangs zwischen Fernsehberichterstattung und zunehmend distanzierteren Bevölkerungseinstellungen gegenüber Staat und Politik („Videomalaise“) stehen die Massenmedien im Verdacht, für die Erosion der politischen Unterstützung mitverantwortlich zu sein. Zahlreiche Untersuchungen belegen dies bereits, aber Daniela Floß bereichert das Forschungsfeld durch den psychologischen Blickwinkel, den sie in ihrer Dissertationsschrift einnimmt: Sie interessiert sich für die Mechanismen, die bewirken, dass sich für die Politik wenig schmeichelhafte Medienberichte in skeptischen Einstellungen gegenüber der Demokratie, ihren Institutionen und zentralen Akteuren niederschlagen.

Nach der Darstellung des Forschungsstandes - insbesondere im Hinblick auf solche Mechanismen - entwickelt sie ein Modell, in dem das Zusammenspiel von individuellen Erwartungen an den Ablauf und die Ergebnisse politischer Prozesse und individuellen Wahrnehmungen, wie Politik tatsächlich funktioniert beziehungsweise was sie leisten sollte, im Mittelpunkt steht. Das Argument ist einfach: Medien nehmen durch die Art und Weise der Berichterstattung Einfluss auf die individuelle Wahrnehmung von politischen Prozessen. Diese Wahrnehmungen können sich in politischen Einstellungen niederschlagen. Wie stark dieser Einfluss ausfällt, hängt davon ab, wie deutlich sich die Wahrnehmungen von unseren Erwartungen an den Ablauf beziehungsweise das Ergebnis politischer Prozesse unterschei- 